MZ-TH/01-32

hep-th/0311075

October 2003

\title{
Explicitly symmetrical treatment of three-body phase space
}

\author{
A. I. Davydychev ${ }^{a, b, c, *}$ and R. Delbourgo ${ }^{a, \dagger}$ \\ a School of Mathematics and Physics, University of Tasmania, \\ GPO Box 252-21, Hobart, Tasmania 7001, Australia \\ ${ }^{b}$ Institute for Nuclear Physics, Moscow State University, \\ 119992 Moscow, Russia \\ ${ }^{c}$ Department of Physics, University of Mainz, \\ Staudingerweg 7, D-55099 Mainz, Germany
}

\begin{abstract}
We derive expressions for three-body phase space that are explicitly symmetrical in the masses of the three particles. We study geometrical properties of the variables involved in elliptic integrals and demonstrate that it is convenient to use the Jacobian zeta function to express the results in four and six dimensions.
\end{abstract}

${ }^{*}$ Present address: Schlumberger, SPC, 155 Industrial Dr., Sugar Land, TX 77478, USA.

Email address: davyd@thep.physik.uni-mainz.de

${ }^{\dagger}$ Email address: bob.delbourgo@utas.edu.au 


\section{Introduction}

The subject of three-body (and, in general, $N$-body) relativistic phase space is as old as the hills and one might well think that all that there is to know is known about it. In numerical and experimental terms this is indeed true: for a long time Dalitz plots [1, 2] have been routinely used in picturing data and they prove extremely helpful for picking out resonant intermediate states of particular spin by their preferential population of the plots. In the absence of any amplitude modulation by resonances or otherwise, the plots are at their blandest as they just represent three-body phase space.

One of the first comprehensive references on this subject is the paper by Almgren [3]. In his normalization, the integral over the $N$-particle phase space is defined as

$$
I_{N}^{(D)}\left(p, m_{1}, \ldots, m_{N}\right)=\int \cdots \int\left\{\prod_{i=1}^{N} \mathrm{~d}^{D} p_{i} \delta\left(p_{i}^{2}-m_{i}^{2}\right) \theta\left(p_{i}^{0}\right)\right\} \delta\left(\sum_{i=1}^{N} p_{i}-p\right)
$$

where $p$ is the total momentum. From now on, we will frequently use the notation $p=\sqrt{p^{2}}$, since usually it is easy to distinguish it from the cases when the four-dimensional vector $p$ is meant. As a rule, we will also omit the arguments of $I_{N}^{(D)}$. In four dimensions $(D=4)$, we will denote $I_{N} \equiv I_{N}^{(4)}$ (this is the original Almgren's notation). More details about integrals in other dimensions can be found in Ref. 4] and in the rest of this paper. Worth mentioning is that $I_{N}^{(D)}$ is easy to work out for odd values of $D$, whereas considering even values of $D$ brings in elliptic functions and is more difficult.

For kinematical reasons, it is clear that the results for the integrals (1.1) have no physical meaning if the absolute value of the momentum $p$ is less than the sum of the masses. Therefore, in what follows we will imply that all results for $I_{N}^{(D)}$ are accompanied by $\theta\left\{p^{2}-\left(m_{1}+\ldots+m_{N}\right)^{2}\right\}$, without writing this theta function explicitly. In Refs. [5, 3], integral recurrence relations for $I_{N}($ at $D=4)$ were discussed. For an arbitrary dimension $D$, the generating relation can be presented as

$$
I_{N}^{(D)}\left(p, m_{1}, \ldots, m_{N}\right)=\int \mathrm{d} s I_{R+1}^{(D)}\left(p, \sqrt{s}, m_{N-R+1}, \ldots, m_{N}\right) I_{N-R}^{(D)}\left(\sqrt{s}, m_{1}, \ldots, m_{N-R}\right) .
$$

Taking into account the theta functions associated with $I_{N-R}^{(D)}$ and $I_{R+1}^{(D)}$, one can see that the actual limits of the integration variable $s$ in Eq. (1.2) extend from $\left(\sum_{i=1}^{N-R} m_{i}\right)^{2}$ to $\left(p-\sum_{i=N-R+1}^{N} m_{i}\right)^{2}$. Another type of integral recurrence relations for $I_{N}^{(D)}$, with respect to the value of $D$, was considered in Ref. [6].

The simplest example is the two-particle phase space, $N=2$. In this case, the phasespace integral (1.1) in four dimensions can be easily evaluated as

$$
I_{2}=\frac{\pi}{2 p^{2}} \sqrt{\lambda\left(p^{2}, m_{1}^{2}, m_{2}^{2}\right)}
$$

where

$$
\lambda(x, y, z) \equiv x^{2}+y^{2}+z^{2}-2 x y-2 y z-2 z x
$$


is nothing but the well-known Källen function [7].

Using Eqs. (1.2) and (1.3) (for the case $D=4, R=1$ ), one can obtain the following integral representation [3, 8] for the three-particle $(N=3)$ phase space:

$$
I_{3}=\frac{\pi^{2}}{4 p^{2}} \int_{s_{2}}^{s_{3}} \frac{\mathrm{d} s}{s} \sqrt{\left(s-s_{1}\right)\left(s-s_{2}\right)\left(s_{3}-s\right)\left(s_{4}-s\right)},
$$

with

$$
s_{1}=\left(m_{1}-m_{2}\right)^{2}, \quad s_{2}=\left(m_{1}+m_{2}\right)^{2}, \quad s_{3}=\left(p-m_{3}\right)^{2}, \quad s_{4}=\left(p+m_{3}\right)^{2},
$$

so that $s_{1} \leq s_{2} \leq s_{3} \leq s_{4}$. The result of the calculation of the integral (1.5) can be expressed in terms of the elliptic integrals [3, 8] (for convenience, we collect the definitions and relevant properties of elliptic integrals in an Appendix),

$$
\begin{aligned}
I_{3}= & \frac{\pi^{2}}{4 p^{2} \sqrt{Q_{+}}}\left\{\frac{1}{2} Q_{+}\left(m_{1}^{2}+m_{2}^{2}+m_{3}^{2}+p^{2}\right) E(k)\right. \\
& +4 m_{1} m_{2}\left[\left(p-m_{3}\right)^{2}-\left(m_{1}-m_{2}\right)^{2}\right]\left[\left(p+m_{3}\right)^{2}-m_{3} p+m_{1} m_{2}\right] K(k) \\
& +8 m_{1} m_{2}\left[\left(m_{1}^{2}+m_{2}^{2}\right)\left(p^{2}+m_{3}^{2}\right)-2 m_{1}^{2} m_{2}^{2}-2 m_{3}^{2} p^{2}\right] \Pi\left(\alpha_{1}^{2}, k\right) \\
& \left.-8 m_{1} m_{2}\left(p^{2}-m_{3}^{2}\right)^{2} \Pi\left(\alpha_{2}^{2}, k\right)\right\}
\end{aligned}
$$

where we use the following notations:

$$
\begin{aligned}
Q_{+} & \equiv\left(p+m_{1}+m_{2}+m_{3}\right)\left(p+m_{1}-m_{2}-m_{3}\right)\left(p-m_{1}+m_{2}-m_{3}\right)\left(p-m_{1}-m_{2}+m_{3}\right), \\
Q_{-} & \equiv\left(p-m_{1}-m_{2}-m_{3}\right)\left(p-m_{1}+m_{2}+m_{3}\right)\left(p+m_{1}-m_{2}+m_{3}\right)\left(p+m_{1}+m_{2}-m_{3}\right), \\
k & \equiv \sqrt{\frac{Q_{-}}{Q_{+}}}, \quad \alpha_{1}^{2}=\frac{\left(p-m_{3}\right)^{2}-\left(m_{1}+m_{2}\right)^{2}}{\left(p-m_{3}\right)^{2}-\left(m_{1}-m_{2}\right)^{2}}, \quad \alpha_{2}^{2}=\frac{\left(m_{1}-m_{2}\right)^{2}}{\left(m_{1}+m_{2}\right)^{2}} \alpha_{1}^{2} .
\end{aligned}
$$

We note that in Ref. [8] the notation $q_{ \pm \pm} \equiv\left(p \pm m_{3}\right)^{2}-\left(m_{1} \pm m_{2}\right)^{2}$ was used. In particular, we have $Q_{+}=q_{++} q_{--}, Q_{-}=q_{+-} q_{-+}, k^{2}=q_{+-} q_{-+} /\left(q_{++} q_{--}\right), \alpha_{1}^{2}=q_{-+} / q_{--}$. Note that $Q_{ \pm}$differ by the sign of $p$ only.

It is clear from the definition (1.1) that $I_{3}$ should be a symmetrical function of the three masses $m_{1}, m_{2}, m_{3}$. The representation (1.7) in terms of elliptic integrals is however not explicitly symmetrical in the masses, although it must be implicitly so. One may of course generate a symmetrical form by averaging the unsymmetrical-looking expressions over the three possible permutations of $m_{i}$, but this would be "cheating" since each one of them should be symmetrical by itself, although this is hardly transparent.

Note that the quantities $Q_{+}$and $Q_{-}$(and, therefore, the argument $k$ ) are totally symmetric in $m_{1}, m_{2}, m_{3}$. (In fact, they are symmetric in all four arguments $p, m_{1}, m_{2}, m_{3}$.) Therefore, the term containing $E(k)$ in Eq. (1.7) is also symmetric. The function $K(k)$ 
itself is also symmetric, but its coefficient is not symmetric. We also note that the product of $Q_{+}$and $Q_{-}$produces the quantity

$$
\begin{aligned}
D_{123} \equiv Q_{+} Q_{-}= & {\left[p^{2}-\left(m_{1}+m_{2}+m_{3}\right)^{2}\right]\left[p^{2}-\left(-m_{1}+m_{2}+m_{3}\right)^{2}\right] } \\
& \times\left[p^{2}-\left(m_{1}-m_{2}+m_{3}\right)^{2}\right]\left[p^{2}-\left(m_{1}+m_{2}-m_{3}\right)^{2}\right]
\end{aligned}
$$

that occurs in recurrence relations for the sunset diagram (see, e.g., in [9, 10]). It should be noted that the imaginary part of the sunset diagram is proportional to the three-particle phase-space integral. For instance, in the notation of Ref. [8], $\operatorname{Im}\left(T_{123}\right)=-4 \pi^{-1} I_{3}$. We also note that $\rho_{N}^{D}$ considered in Ref. [4, 6] are related to $I_{N}^{(D)}$ as $\rho_{N}^{D}=(2 \pi)^{N+D-N D} I_{N}^{(D)}$.

For equal masses, $m_{1}=m_{2}=m_{3} \equiv m$, Eq. (1.7) yields

$$
\frac{\pi^{2}}{4 p^{2}} \sqrt{(p-m)(p+3 m)}\left\{\frac{1}{2}(p-m)\left(p^{2}+3 m^{2}\right) E\left(k_{\mathrm{eq}}\right)-4 m^{2} p K\left(k_{\mathrm{eq}}\right)\right\},
$$

with

$$
k_{\mathrm{eq}}=\sqrt{\frac{(p+m)^{3}(p-3 m)}{(p-m)^{3}(p+3 m)}} .
$$

Some other special cases of Eq. (1.7) are described in Refs. [8, 11.

This paper is devoted to a new way of exhibiting the results in an explicitly symmetrical manner. To do this, we will employ another integral representation for $I_{3}$, in terms of Mandelstam variables $s, t, u[12$ and the Kibble cubic $\Phi(s, t, u)$ [13. In particular, we will show that it is convenient to present the result (1.7) in terms of Jacobi $Z$ function whose definition is given in the Appendix.

\section{Phase space integrals}

As an illustration, let us demonstrate how the connection with the Dalitz picture can be derived directly from the definition (1.1). The $D$-dimensional vector $p$ can be presented as $\left(p^{0}, \mathbf{p}\right)$, where $\mathbf{p}$ is the $(D-1)$-dimensional Euclidean vector of space components. Without loss of generality, we can work in the center-of-mass frame, $p=\left(p^{0}, \mathbf{0}\right)$. Using the integral representation

$$
\delta\left(\sum_{i=1}^{N} p_{i}-p\right)=\frac{1}{(2 \pi)^{D}} \int \mathrm{d}^{D} x \exp \left\{\mathrm{i} \sum_{i=1}^{N}\left(p_{i} x\right)-\mathrm{i}(p x)\right\},
$$

with $(p x)=p^{0} x^{0}$, we get

$$
I_{N}^{(D)}=\frac{1}{(2 \pi)^{D}} \int \mathrm{d}^{D} x e^{-\mathrm{i} p^{0} x^{0}}\left\{\prod_{i=1}^{N} \int \mathrm{d}^{D} p_{i} \delta\left(p_{i}^{2}-m_{i}^{2}\right) \theta\left(p_{i}^{0}\right) e^{\mathrm{i}\left(p_{i} x\right)}\right\} .
$$


(Similar method was used in Ref. [14.) Integrating over $(D-1)$-dimensional angles of $\mathbf{p}_{i}$ we get

$$
\int \mathrm{d}^{D} p_{i} \delta\left(p_{i}^{2}-m_{i}^{2}\right) \theta\left(p_{i}^{0}\right) e^{\mathrm{i}\left(p_{i} x\right)}=\frac{(2 \pi)^{(D-1) / 2}}{2 \xi^{(D-3) / 2}} \int_{0}^{\infty} \frac{\rho_{i}^{(D-1) / 2} \mathrm{~d} \rho_{i}}{\sqrt{\rho_{i}^{2}+m_{i}^{2}}} J_{(D-3) / 2}\left(\rho_{i} \xi\right) e^{\mathrm{i} x^{0} \sqrt{\rho_{i}^{2}+m_{i}^{2}}},
$$

with $\rho_{i} \equiv\left|\mathbf{p}_{i}\right|$ and $\xi \equiv|\mathbf{x}|$. In the four-dimensional case the Bessel function reduces to an elementary function, $J_{1 / 2}\left(\rho_{i} \xi\right)=\left[2 /\left(\pi \rho_{i} \xi\right)\right]^{1 / 2} \sin \left(\rho_{i} \xi\right)$. We note an analogy with the calculation of Feynman integrals in the coordinate space [15, 22, when each massive propagator yields a (modified) Bessel function.

Let us consider, for example, the two-particle phase space. Then, the integration over $\xi$ gives us

$$
\int_{0}^{\infty} \xi \mathrm{d} \xi J_{\nu}\left(\rho_{1} \xi\right) J_{\nu}\left(\rho_{2} \xi\right)=2 \delta\left(\rho_{1}^{2}-\rho_{2}^{2}\right)
$$

with $\nu=(D-3) / 2$, so that we can put $\rho_{1}=\rho_{2} \equiv \rho$, whereas the integration over $x^{0}$ yields another delta function, $\delta\left(p-\sqrt{\rho^{2}+m_{1}^{2}}-\sqrt{\rho^{2}+m_{2}^{2}}\right)$ in the center-of-mass frame. The resulting integral

$$
I_{2}^{(D)}=\frac{\pi^{(D-1) / 2}}{2 \Gamma\left(\frac{D-1}{2}\right)} \int_{0}^{\infty} \frac{\rho^{D-2} \mathrm{~d} \rho}{\sqrt{\rho^{2}+m_{1}^{2}} \sqrt{\rho^{2}+m_{2}^{2}}} \delta\left(p-\sqrt{\rho^{2}+m_{1}^{2}}-\sqrt{\rho^{2}+m_{2}^{2}}\right)
$$

can be easily evaluated, yielding (see, e.g., in Ref. [6])

$$
I_{2}^{(D)}=\frac{\pi^{(D-1) / 2}}{(2 p)^{D-2} \Gamma\left(\frac{D-1}{2}\right)}\left[\lambda\left(p^{2}, m_{1}^{2}, m_{2}^{2}\right)\right]^{(D-3) / 2},
$$

where $\lambda$ is defined in Eq. (1.4). For $D=4$, Eq. (2.5) reduces to the well-known answer (1.3).

For the three-particle phase-space integral we get

$$
I_{3}^{(D)}=\frac{2^{(D-7) / 2} \pi^{D-2}}{\Gamma\left(\frac{D-1}{2}\right)} \int_{0}^{\infty} \frac{\mathrm{d} \xi}{\xi^{(D-5) / 2}} \int_{-\infty}^{\infty} \mathrm{d} x^{0} e^{-\mathrm{i} p^{0} x^{0}} \prod_{i=1}^{3} \int_{0}^{\infty} \frac{\rho_{i}^{(D-1) / 2} \mathrm{~d} \rho_{i}}{\sqrt{\rho_{i}^{2}+m_{i}^{2}}} J_{(D-3) / 2}\left(\rho_{i} \xi\right) e^{\mathrm{i} x^{0} \sqrt{\rho_{i}^{2}+m_{i}^{2}}}
$$

Here we can integrate over $\xi$, using (see Ref. [16])

$$
\int_{0}^{\infty} \frac{\mathrm{d} \xi}{\xi^{\nu-1}} J_{\nu}\left(\rho_{1} \xi\right) J_{\nu}\left(\rho_{2} \xi\right) J_{\nu}\left(\rho_{3} \xi\right)=\frac{2 \theta\left\{-\lambda\left(\rho_{1}^{2}, \rho_{2}^{2}, \rho_{3}^{2}\right)\right\}\left[-\lambda\left(\rho_{1}^{2}, \rho_{2}^{2}, \rho_{3}^{2}\right)\right]^{\nu-1 / 2}}{\pi^{1 / 2} \Gamma\left(\nu+\frac{1}{2}\right)\left(8 \rho_{1} \rho_{2} \rho_{3}\right)^{\nu}}
$$

(with $\nu=(D-3) / 2$ ), where $\lambda$ is the Källen function (1.4). In fact, in our case, when all $\rho_{i} \geq 0$,

$$
\theta\left\{-\lambda\left(\rho_{1}^{2}, \rho_{2}^{2}, \rho_{3}^{2}\right)\right\}=\theta\left(\rho_{1}+\rho_{2}-\rho_{3}\right) \theta\left(\rho_{2}+\rho_{3}-\rho_{1}\right) \theta\left(\rho_{3}+\rho_{1}-\rho_{2}\right)
$$


i.e., it equals 1 when one can compose a triangle with sides $\rho_{1}, \rho_{2}, \rho_{3}$, and gives 0 otherwise (cf. Eq. (11) of Ref. [17]).

Introducing notation $\sigma_{i}=\sqrt{\rho_{i}^{2}+m_{i}^{2}}$ and integrating over $x^{0}$ (getting a $\delta$ function) we arrive at

$$
\begin{aligned}
I_{3}^{(D)}= & \frac{\pi^{D-2}}{\Gamma(D-2)} \int_{m_{1}}^{\infty} \int_{m_{2}}^{\infty} \int_{m_{3}}^{\infty} \mathrm{d} \sigma_{1} \mathrm{~d} \sigma_{2} \mathrm{~d} \sigma_{3} \delta\left(p-\sigma_{1}-\sigma_{2}-\sigma_{3}\right) \\
& \times\left[-\lambda\left(\sigma_{1}^{2}-m_{1}^{2}, \sigma_{2}^{2}-m_{2}^{2}, \sigma_{3}^{2}-m_{3}^{2}\right)\right]^{(D-4) / 2} \theta\left\{-\lambda\left(\sigma_{1}^{2}-m_{1}^{2}, \sigma_{2}^{2}-m_{2}^{2}, \sigma_{3}^{2}-m_{3}^{2}\right)\right\}
\end{aligned}
$$

In four dimensions the factor $[-\lambda]^{(D-4) / 2}$ disappears and, geometrically, we need to calculate a closed area on the plane $\sigma_{1}+\sigma_{2}+\sigma_{3}=p^{0} \equiv p$, with the boundary of the figure described by

$$
\lambda\left(\sigma_{1}^{2}-m_{1}^{2}, \sigma_{2}^{2}-m_{2}^{2}, \sigma_{3}^{2}-m_{3}^{2}\right)=0, \quad \sigma_{1}+\sigma_{2}+\sigma_{3}=p .
$$

Furthermore, introducing Mandelstam-type variables

$$
s=p^{2}+m_{3}^{2}-2 p \sigma_{3}, \quad t=p^{2}+m_{1}^{2}-2 p \sigma_{1}, \quad u=p^{2}+m_{2}^{2}-2 p \sigma_{2}
$$

satisfying

$$
s+t+u=m_{1}^{2}+m_{2}^{2}+m_{3}^{2}+p^{2} \equiv w_{0},
$$

one arrives at another integral representation (the limits of integration are discussed below),

$I_{3}^{(D)}=\frac{\pi^{D-2}}{4 p^{D-2} \Gamma(D-2)} \iiint \mathrm{d} s \mathrm{~d} t \mathrm{~d} u \delta\left(s+t+u-w_{0}\right)[\Phi(s, t, u)]^{(D-4) / 2} \theta\{\Phi(s, t, u)\}$,

where

$$
\Phi(s, t, u)=-\frac{1}{16 p^{2}} \lambda\left\{\lambda\left(s, m_{3}^{2}, p^{2}\right), \lambda\left(t, m_{1}^{2}, p^{2}\right), \lambda\left(u, m_{2}^{2}, p^{2}\right)\right\}
$$

can also be written in a more familiar Kibble cubic form [13],

$$
\begin{aligned}
\Phi(s, t, u)= & s t u-s\left(m_{1}^{2} m_{2}^{2}+p^{2} m_{3}^{2}\right)-t\left(m_{2}^{2} m_{3}^{2}+p^{2} m_{1}^{2}\right)-u\left(m_{3}^{2} m_{1}^{2}+p^{2} m_{2}^{2}\right) \\
& +2\left(m_{1}^{2} m_{2}^{2} m_{3}^{2}+p^{2} m_{1}^{2} m_{2}^{2}+p^{2} m_{2}^{2} m_{3}^{2}+p^{2} m_{3}^{2} m_{1}^{2}\right),
\end{aligned}
$$

provided that the condition (2.12) is satisfied. In particular, in four dimensions we have

$$
I_{3}=\frac{\pi^{2}}{4 p^{2}} \iiint \mathrm{d} s \mathrm{~d} t \mathrm{~d} u \delta\left(s+t+u-w_{0}\right) \theta\{\Phi(s, t, u)\}
$$

According to the definition (2.11) in terms of $\sigma_{i}$, one can see that the maximal values of $s, t$ and $u$ (corresponding to the upper limits of integration in Eqs. (2.13) and (2.16) are $s_{\max }=\left(p-m_{3}\right)^{2}, t_{\max }=\left(p-m_{1}\right)^{2}$ and $u_{\max }=\left(p-m_{2}\right)^{2}$. To define the minimal 


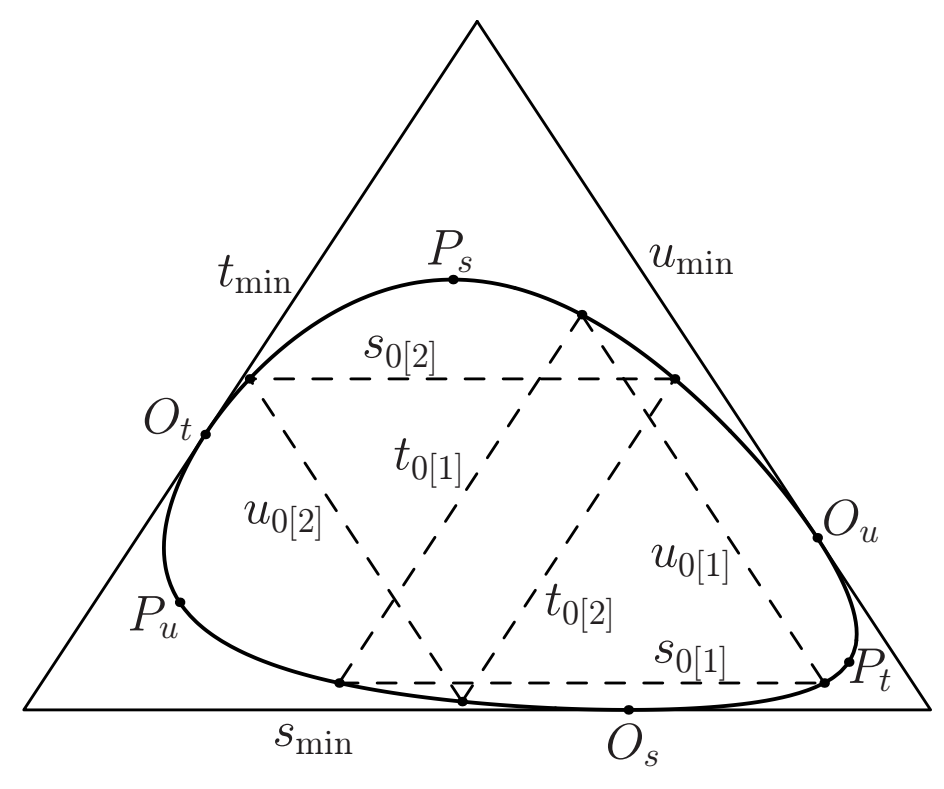

Figure 1: The Dalitz-Kibble integration area

values of $s, t$ and $u$, the familiar Dalitz-Kibble picture given in Fig. 1 is useful. Due to the condition (2.12), the region of integration is restricted by a triangle, with $s \geq s_{\min }=$ $\left(m_{1}+m_{2}\right)^{2}, t \geq t_{\min }=\left(m_{2}+m_{3}\right)^{2}$ and $u \geq u_{\min }=\left(m_{1}+m_{3}\right)^{2}$. Moreover, due to the theta function $\theta\{\Phi(s, t, u)\}$ the region of integration is in fact restricted by the interior of the cubic curve $\Phi(s, t, u)=0$, see Fig. 1 . Within that area, the Mandelstam variables $s, t$ and $u$ take their minimal values in points $O_{s}, O_{t}$ and $O_{u}$, respectively, whereas their maximal values correspond to the points $P_{s}, P_{t}$ and $P_{u}$. (The dashed triangles will be discussed below in Section 4.)

The function $\Phi(s, t, u)$ has a maximum within the region of integration. For equal masses, the maximal value $\Phi_{\max }=\frac{1}{27} p^{2}\left(p^{2}-9 m^{2}\right)^{2}$ occurs at $s=t=u=\frac{1}{3}\left(p^{2}+3 m^{2}\right)$. For the general unequal masses, one needs to solve a fourth-order algebraic equation to find the position of the maximum.

We note that the representation (2.14) can be extracted from Eq. (5.39) of [18, using symmetry properties. Our $\Phi(s, t, u)$ corresponds to $-G\left(s, t, p^{2}, m_{2}^{2}, m_{1}^{2}, m_{3}^{2}\right)$, in the notations of [18. The $G$-function is symmetric with respect to the permutations of three pairs of arguments, $(s, t),\left(p^{2}, m_{2}^{2}\right)$ and $\left(m_{1}^{2}, m_{3}^{2}\right)$. Although the authors presume from their Eq. (5.39) that "from a practical point of view this identity is not very useful", we found that its symmetric form is certainly helpful in understanding the structural properties of phase space integrals. 


\section{Geometrical interpretation}

Let us introduce

$$
c_{12}=\frac{s-m_{1}^{2}-m_{2}^{2}}{2 m_{1} m_{2}}, \quad c_{23}=\frac{t-m_{2}^{2}-m_{3}^{2}}{2 m_{2} m_{3}}, \quad c_{13}=\frac{u-m_{1}^{2}-m_{3}^{2}}{2 m_{1} m_{3}} .
$$

Then, the function $\Phi(s, t, u)$ can be presented as a Gram determinant,

$$
\Phi(s, t, u)=4 m_{1}^{2} m_{2}^{2} m_{3}^{2}\left|\begin{array}{ccc}
1 & c_{12} & c_{13} \\
c_{12} & 1 & c_{23} \\
c_{13} & c_{23} & 1
\end{array}\right|
$$

whereas the $\delta$ function becomes

$$
\begin{aligned}
\delta(s+t+u & \left.-m_{1}^{2}-m_{2}^{2}-m_{3}^{2}-p^{2}\right) \\
& \Rightarrow \delta\left(m_{1}^{2}+m_{2}^{2}+m_{3}^{2}+2 m_{1} m_{2} c_{12}+2 m_{2} m_{3} c_{23}+2 m_{1} m_{3} c_{13}-p^{2}\right) .
\end{aligned}
$$

In this way, we get

$$
\begin{aligned}
I_{3}= & \frac{2 \pi^{2}}{p^{2}} m_{1}^{2} m_{2}^{2} m_{3}^{2} \iiint \mathrm{d} c_{12} \mathrm{~d} c_{13} \mathrm{~d} c_{23} \theta\left(\left|\begin{array}{ccc}
1 & c_{12} & c_{13} \\
c_{12} & 1 & c_{23} \\
c_{13} & c_{23} & 1
\end{array}\right|\right) \\
& \times \delta\left(m_{1}^{2}+m_{2}^{2}+m_{3}^{2}+2 m_{1} m_{2} c_{12}+2 m_{2} m_{3} c_{23}+2 m_{1} m_{3} c_{13}-p^{2}\right),
\end{aligned}
$$

where the integration extends over $c_{j l} \geq 1$.

If one were to interpret $c_{j l}$ as the cosines of the angles between the $m_{j}$ and $m_{l}$ sides of a vertex of a parallelepiped (formed by $m_{1}, m_{2}$ and $m_{3}$, see Fig. 2), then all these quantities would have a straightforward geometrical interpretation. Namely, $\Phi(s, t, u)$ would be $4\{\text { volume of parallelepiped }\}^{2}$, whereas the $\delta$ function would tell us that the "principal" diagonal of this parallelepiped should be equal to $p$. In this case, the quantities $\sqrt{s}, \sqrt{t}$ and $\sqrt{u}$ could be identified as the diagonals of the faces of the parallelepiped, see in Fig. 2. Moreover,

$$
\frac{p^{2}+m_{1}^{2}-t}{2 p m_{1}}, \quad \frac{p^{2}+m_{2}^{2}-u}{2 p m_{2}} \text { and } \frac{p^{2}+m_{3}^{2}-s}{2 p m_{3}}
$$

could be understood as cosines of the angles between the diagonal $p$ and the $m_{i}$ sides of the parallelepiped. In other words, these are the angles between $p$ and $m_{i}$ in triangles with sides $\left(p, m_{1}, \sqrt{t}\right),\left(p, m_{2}, \sqrt{u}\right)$ and $\left(p, m_{3}, \sqrt{s}\right)$, respectively.

Using this geometrical picture, we can mention a rather interesting geometrical meaning of Eq. (2.14). Namely, it tells us that the volume of the parallelepiped is $(8 / p)$ times the area of triangle whose sides are given by the areas of triangles formed out of the principal diagonal $p$, one of the face diagonals $(\sqrt{s}, \sqrt{t}$ or $\sqrt{u})$, and the appropriate $m_{3}$, $m_{1}$ or $m_{2}$ side.

However, when we are above the threshold, $p^{2}>\left(m_{1}+m_{2}+m_{3}\right)^{2}$, the quantities $c_{j l}$ exceed one and therefore the expressions should be understood in the sense of analytic 


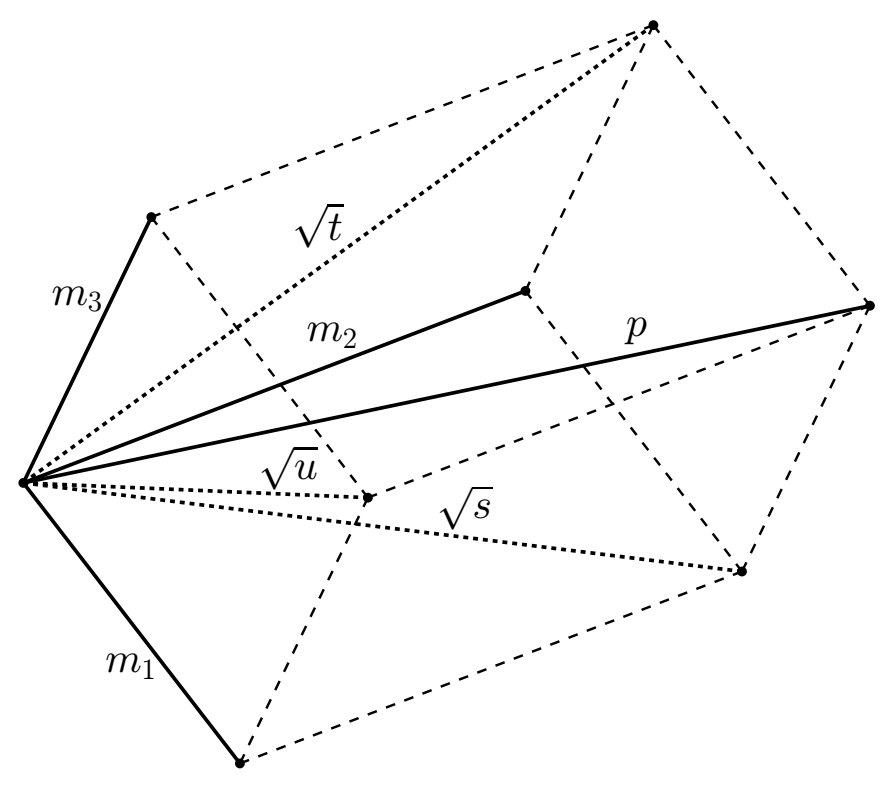

Figure 2: The parallelepiped interpretation

continuation, i.e. as hyperbolic cosines. The same is valid for the triangles $\left(p, m_{3}, \sqrt{s}\right)$, etc.: they should also be understood in the sense of analytic continuation, since $p \geq m_{3}+\sqrt{s}$, etc. Therefore, the quantities $\sigma_{i} / m_{i}$ should also be understood as hyperbolic cosines, whereas $\sqrt{\sigma_{i}^{2}-m_{i}^{2}} / m_{i}$ are hyperbolic sines.

Nevertheless, in the region below the threshold (which we need, for instance, to describe the real part of the sunset diagram), this geometrical picture can have direct meaning, generalizing the picture we had for the one-loop two-point function [19].

\section{Kibble cubic characteristics}

Suppose

$$
\left(s_{0}, t_{0}, w_{0}-s_{0}-t_{0}\right), \quad\left(s_{0}, w_{0}-s_{0}-u_{0}, u_{0}\right), \quad\left(w_{0}-t_{0}-u_{0}, t_{0}, u_{0}\right)
$$

all are the roots of the equation $\Phi(s, t, u)=0$. Then, we can present $\Phi(s, t, u)$ as

$$
\Phi(s, t, u)=s t u-s t_{0} u_{0}-s_{0} t u_{0}-s_{0} t_{0} u+2 s_{0} t_{0} u_{0} .
$$

Furthermore, if we shift the Mandelstam variables as

$$
s=s_{0}+s^{\prime}, \quad t=t_{0}+t^{\prime}, \quad u=u_{0}+u^{\prime},
$$

subject to the condition

$$
s^{\prime}+t^{\prime}+u^{\prime}=w_{0}-s_{0}-t_{0}-u_{0} \equiv w_{0}^{\prime},
$$


then

$$
\Phi(s, t, u) \Rightarrow s^{\prime} t^{\prime} u^{\prime}+s_{0} t^{\prime} u^{\prime}+s^{\prime} t_{0} u^{\prime}+s^{\prime} t^{\prime} u_{0} .
$$

Using equation Eq. (4.2) and defining

$$
c_{t u} \equiv \sqrt{\frac{t_{0} u_{0}}{t u}}, \quad c_{s t} \equiv \sqrt{\frac{s_{0} t_{0}}{s t}}, \quad c_{s u} \equiv \sqrt{\frac{s_{0} u_{0}}{s u}},
$$

we arrive at another Gram determinant representation for $\Phi(s, t, u)$ (cf. Eq. (3.2)),

$$
\Phi(s, t, u)=s t u\left|\begin{array}{ccc}
1 & c_{t u} & c_{s t} \\
c_{t u} & 1 & c_{s u} \\
c_{s t} & c_{s u} & 1
\end{array}\right|
$$

There are (at least) two sets of solutions (4.1) that can be described as

$$
s_{0}=\frac{A_{1} A_{2}}{A_{3}}, \quad t_{0}=\frac{A_{2} A_{3}}{A_{1}}, \quad u_{0}=\frac{A_{1} A_{3}}{A_{2}},
$$

so that Eq. (4.2) yields

$$
\Phi(s, t, u)=s t u-A_{1}^{2} t-A_{2}^{2} u-A_{3}^{2} s+2 A_{1} A_{2} A_{3} .
$$

The first set of solutions corresponds to

$$
A_{1} \equiv p m_{1}+m_{2} m_{3}, \quad A_{2} \equiv p m_{2}+m_{3} m_{1}, \quad A_{3} \equiv p m_{3}+m_{1} m_{2} .
$$

For this set, we have

$$
\begin{aligned}
w_{0}^{\prime} & =w_{0}-s_{0}-t_{0}-u_{0}=\frac{m_{1} m_{2} m_{3} p Q_{-}}{A_{1} A_{2} A_{3}}, \\
c_{t u} & =\frac{p m_{3}+m_{1} m_{2}}{\sqrt{t u}}, \quad c_{s t}=\frac{p m_{2}+m_{1} m_{3}}{\sqrt{s t}}, \quad c_{s u}=\frac{p m_{1}+m_{2} m_{3}}{\sqrt{s u}} .
\end{aligned}
$$

Note that if we change $p \rightarrow-p$ in Eq. (4.10), this would also be a solution, which would correspond to a "non-physical" branch of the Kibble cubic.

The second set of solutions corresponds to

$$
A_{1} \equiv \frac{1}{2}\left(p^{2}+m_{1}^{2}-m_{2}^{2}-m_{3}^{2}\right), \quad A_{2} \equiv \frac{1}{2}\left(p^{2}-m_{1}^{2}+m_{2}^{2}-m_{3}^{2}\right), \quad A_{3} \equiv \frac{1}{2}\left(p^{2}-m_{1}^{2}-m_{2}^{2}+m_{3}^{2}\right) \text {. }
$$

For this set, we get

$$
\begin{aligned}
& w_{0}^{\prime}=w_{0}-s_{0}-t_{0}-u_{0}=-\frac{Q_{+} Q_{-}}{16 A_{1} A_{2} A_{3}}=-\frac{D_{123}}{16 A_{1} A_{2} A_{3}}, \\
& c_{t u}=\frac{p^{2}-m_{1}^{2}-m_{2}^{2}+m_{3}^{2}}{2 \sqrt{t u}}, \quad c_{s t}=\frac{p^{2}-m_{1}^{2}+m_{2}^{2}-m_{3}^{2}}{2 \sqrt{s t}}, \quad c_{s u}=\frac{p^{2}+m_{1}^{2}-m_{2}^{2}-m_{3}^{2}}{2 \sqrt{s u}} .
\end{aligned}
$$


It should be noted that the value of $s_{0}$ corresponding to the second set satisfies

$$
\lambda\left(s_{0}, m_{1}^{2}, m_{2}^{2}\right)=\lambda\left(s_{0}, p^{2}, m_{3}^{2}\right),
$$

i.e., the areas (or their analytical continuations) of triangles with sides $\left(\sqrt{s_{0}}, m_{1}, m_{2}\right)$ and $\left(\sqrt{s_{0}}, p, m_{3}\right)$ are equal. Analogously,

$$
\lambda\left(t_{0}, m_{2}^{2}, m_{3}^{2}\right)=\lambda\left(t_{0}, p^{2}, m_{1}^{2}\right), \quad \lambda\left(u_{0}, m_{1}^{2}, m_{3}^{2}\right)=\lambda\left(u_{0}, p^{2}, m_{2}^{2}\right) .
$$

Moreover, one can get the direct geometrical interpretation of the quantities (4.15) through the familiar parallelepiped picture given in Fig. 2. Namely, $c_{t u}$ is nothing but the cosine between the face diagonals $\sqrt{t}$ and $\sqrt{u}$. Accordingly, $c_{s u}$ is the cosine of the angle between $\sqrt{s}$ and $\sqrt{u}$ diagonals, whilst $c_{s t}$ is the cosine of the angle between $\sqrt{s}$ and $\sqrt{t}$ diagonals. If we construct a tetrahedron using the $\sqrt{s}, \sqrt{t}$ and $\sqrt{u}$ diagonals then, according to Eq. (4.7), $\Phi(s, t, u)$ would represent 36 times its volume squared.

In the Dalitz-Kibble plot shown in Fig. 1 we connect the points (4.1) for each of the two sets by dashed lines, introducing subscripts [1] and [2] for the first and the second set, respectively. The two resulting "dashed" triangles indicate that the two sets of solutions are complementary to each other. Namely, the boundary of the Dalitz plot confines the products $t u$, st and $s u$ as follows:

$$
\begin{aligned}
& \left(p m_{3}+m_{1} m_{2}\right)^{2} \leq t u \leq \frac{1}{4}\left(p^{2}-m_{1}^{2}-m_{2}^{2}+m_{3}^{2}\right)^{2}, \\
& \left(p m_{2}+m_{1} m_{3}\right)^{2} \leq s t \leq \frac{1}{4}\left(p^{2}-m_{1}^{2}+m_{2}^{2}-m_{3}^{2}\right)^{2}, \\
& \left(p m_{1}+m_{2} m_{3}\right)^{2} \leq s u \leq \frac{1}{4}\left(p^{2}+m_{1}^{2}-m_{2}^{2}-m_{3}^{2}\right)^{2},
\end{aligned}
$$

or, equivalently,

$$
\left(t_{0} u_{0}\right)_{[1]} \leq t u \leq\left(t_{0} u_{0}\right)_{[2]}, \quad\left(s_{0} t_{0}\right)_{[1]} \leq s t \leq\left(s_{0} t_{0}\right)_{[2]}, \quad\left(s_{0} u_{0}\right)_{[1]} \leq s u \leq\left(s_{0} u_{0}\right)_{[2]} .
$$

In other words, the first and the second sets yield, respectively, the minimal and the maximal values of $t u$, st and $s u$.

Let us consider the corresponding values of the "cosines" $c_{s u}, c_{s t}$ and $c_{t u}$. For the first set, $c_{s u}, c_{s t}$ and $c_{t u}$ would vary between 1 and $\cos \varphi_{i}(i=1,2,3)$, respectively, where

$$
\cos \varphi_{1}=\frac{2\left(p m_{1}-m_{2} m_{3}\right)}{p^{2}+m_{1}^{2}-m_{2}^{2}-m_{3}^{2}}, \quad \cos \varphi_{2}=\frac{2\left(p m_{2}-m_{3} m_{1}\right)}{p^{2}-m_{1}^{2}+m_{2}^{2}-m_{3}^{2}}, \quad \cos \varphi_{3}=\frac{2\left(p m_{3}-m_{1} m_{2}\right)}{p^{2}-m_{1}^{2}-m_{2}^{2}+m_{3}^{2}} .
$$

For the second set, $c_{s u}, c_{s t}$ and $c_{t u}$ would vary between 1 and $1 / \cos \varphi_{i}$. This means that we need to understand them in the sense of analytic continuation.

The angles $\varphi_{i}$ will be very important below. Their sines can be presented as

$$
\sin \varphi_{1}=\frac{\sqrt{Q_{+}}}{p^{2}+m_{1}^{2}-m_{2}^{2}-m_{3}^{2}}, \quad \sin \varphi_{2}=\frac{\sqrt{Q_{+}}}{p^{2}-m_{1}^{2}+m_{2}^{2}-m_{3}^{2}}, \quad \sin \varphi_{3}=\frac{\sqrt{Q_{+}}}{p^{2}-m_{1}^{2}-m_{2}^{2}+m_{3}^{2}} .
$$


It is interesting that the corresponding Gram determinant can be factorized as

$$
\left|\begin{array}{ccc}
1 & -\cos \varphi_{3} & -\cos \varphi_{2} \\
-\cos \varphi_{3} & 1 & -\cos \varphi_{1} \\
-\cos \varphi_{2} & -\cos \varphi_{1} & 1
\end{array}\right|=\frac{1}{k^{2}} \sin ^{2} \varphi_{1} \sin ^{2} \varphi_{2} \sin ^{2} \varphi_{3}
$$

Eq. (4.22) can be used to express $k$ in terms of $\varphi_{i}$. We also note that

$$
\tan \frac{\varphi_{3}}{2}=\sqrt{\frac{\left(p-m_{3}\right)^{2}-\left(m_{1}-m_{2}\right)^{2}}{\left(p+m_{3}\right)^{2}-\left(m_{1}+m_{2}\right)^{2}}},
$$

and similarly for $\varphi_{1}$ and $\varphi_{2}$. In particular, one can see that at the threshold, $p=m_{1}+$ $m_{2}+m_{3}$, the angles $\varphi_{i}$ are related to the angles $\theta_{i}$ from Eq. (20) of Ref. [20] (see also in Ref. [10]) as $\varphi_{i}=\pi-2 \theta_{i}$, and

$$
\left.\left(\varphi_{1}+\varphi_{2}+\varphi_{3}\right)\right|_{p=m_{1}+m_{2}+m_{3}}=\pi \text {. }
$$

We can also consider associated angles $\psi_{i}$, such that

$$
\sin \psi_{i}=k \sin \varphi_{i}, \quad \cos \psi_{i}=\sqrt{1-k^{2} \sin ^{2} \varphi_{i}}
$$

Explicitly, we get

$$
\sin \psi_{3}=\frac{\sqrt{Q_{-}}}{p^{2}-m_{1}^{2}-m_{2}^{2}+m_{3}^{2}}, \quad \cos \psi_{3}=\frac{2\left(p m_{3}+m_{1} m_{2}\right)}{p^{2}-m_{1}^{2}-m_{2}^{2}+m_{3}^{2}},
$$

etc. For these angles, we get

$$
\left|\begin{array}{ccc}
1 & \cos \psi_{3} & \cos \psi_{2} \\
\cos \psi_{3} & 1 & \cos \psi_{1} \\
\cos \psi_{2} & \cos \psi_{1} & 1
\end{array}\right|=\frac{1}{k^{2}} \sin ^{2} \psi_{1} \sin ^{2} \psi_{2} \sin ^{2} \psi_{3}
$$

\section{A naturally symmetric representation}

Using the representation (4.2) for $\Phi(s, t, u)$, in terms of $s_{0}, t_{0}$ and $u_{0}$, the three-body phase-space integral can be written as

$$
I_{3}=\frac{\pi^{2}}{4 p^{2}} \iiint \mathrm{d} s \mathrm{~d} t \mathrm{~d} u \delta\left(s+t+u-w_{0}\right) \theta\left(s t u-s t_{0} u_{0}-s_{0} t u_{0}-s_{0} t_{0} u+2 s_{0} t_{0} u_{0}\right),
$$

with $w_{0}=p^{2}+m_{1}^{2}+m_{2}^{2}+m_{3}^{2}$. Integrating over $u$ yields

$$
I_{3}=\frac{\pi^{2}}{4 p^{2}} \iint \mathrm{d} s \mathrm{~d} t \theta\left\{\left(s t-s_{0} t_{0}\right)\left(w_{0}-s-t\right)-s t_{0} u_{0}-s_{0} t u_{0}+2 s_{0} t_{0} u_{0}\right\}
$$


Then, integrating over $t$, we basically obtain the difference between the roots of the quadratic argument of the $\theta$ function, which is

$$
\frac{1}{s} \sqrt{s^{4}-2 w_{0} s^{3}+\left(w_{0}^{2}+2 s_{0} t_{0}+2 s_{0} u_{0}-4 t_{0} u_{0}\right) s^{2}-2\left(w_{0} t_{0}+w_{0} u_{0}-4 u_{0} t_{0}\right) s_{0} s+s_{0}^{2}\left(t_{0}-u_{0}\right)^{2}} .
$$

It is easy to check that for both sets of $\left(s_{0}, t_{0}, u_{0}\right)$ the square root takes the familiar form (1.5), which yields the non-symmetric result (1.7) in terms of elliptic integrals.

Starting from the representation (2.13), one can easily generalize the result (1.5) to the $D$-dimensional case as

$$
I_{3}^{(D)}=\frac{\pi^{D-1}}{(4 p)^{D-2} \Gamma^{2}\left(\frac{D-1}{2}\right)} \int_{s_{2}}^{s_{3}} \frac{\mathrm{d} s}{s^{D / 2-1}}\left[\left(s-s_{1}\right)\left(s-s_{2}\right)\left(s_{3}-s\right)\left(s_{4}-s\right)\right]^{(D-3) / 2},
$$

with $s_{i}$ given in Eq. (1.6). Another way to derive the representation (5.3) is to use the recurrence relation (1.2),

$$
I_{3}^{(D)}=\int_{s_{2}}^{s_{3}} \mathrm{~d} s I_{2}^{(D)}\left(p, \sqrt{s}, m_{3}\right) I_{2}^{(D)}\left(\sqrt{s}, m_{1}, m_{2}\right)
$$

and substitite the result (2.5) for $I_{2}^{(D)}$. The result (5.3) corresponds to Eq. (9) of Ref. [4]. [We note that the overall factor on the r.h.s. of Eq. (9) of Ref. [4] should be corrected: $(32 \pi)^{2-2 \ell}$ should be changed into $\frac{1}{2}(16 \pi)^{2-2 \ell}$, with $\left.\ell=D / 2\right]$.

Using representation (5.3), it is easy to see (just substituting $s=x^{2}$ ) that all odddimensional phase-space integrals can be expressed in terms of polynomial functions (see, e.g., in Refs. [21, 22, 23]),

$$
\begin{aligned}
I_{3}^{(3)}= & \frac{\pi^{2}}{2 p}\left(p-m_{1}-m_{2}-m_{3}\right) \\
I_{3}^{(5)}= & \frac{\pi^{4}}{60 p^{3}}\left(p-m_{1}-m_{2}-m_{3}\right)^{3}\left[\frac{1}{7}\left(p-m_{1}-m_{2}-m_{3}\right)^{4}+\left(m_{1}+m_{2}+m_{3}\right) p^{3}\right. \\
& -2\left(m_{1}^{2}+m_{2}^{2}+m_{3}^{2}\right) p^{2}+\left(m_{1}^{3}+m_{2}^{3}+m_{3}^{3}\right) p+12 m_{1} m_{2} m_{3} p \\
& \left.-\left(m_{1}+m_{2}+m_{3}\right)\left(m_{1}+m_{2}\right)\left(m_{2}+m_{3}\right)\left(m_{3}+m_{1}\right)+4 m_{1} m_{2} m_{3}\left(m_{1}+m_{2}+m_{3}\right)\right]
\end{aligned}
$$

etc., which are explicitly symmetric in the masses $m_{i}$. However, the results in even dimensions appear to be less trivial.

It is instructive to consider the two-dimensional case, $D=2$. Then, the integral (5.3) yields just the elliptic integral $K(k)$,

$$
I_{3}^{(2)}=\int_{s_{2}}^{s_{3}} \frac{\mathrm{d} s}{\sqrt{\left(s-s_{1}\right)\left(s-s_{2}\right)\left(s_{3}-s\right)\left(s_{4}-s\right)}}=\frac{2}{\sqrt{Q_{+}}} K(k) .
$$


This is of course explicitly symmetric in the masses without further ado. On the other hand, using the $\delta$ function in Eq. (5.1), we can insert $1=(s+t+u) / w_{0}$ in the integrand, and then consider the three resulting terms (with $s, t$ and $u$ ) separately. In this way, we arrive at an alternative expression,

$$
\begin{aligned}
I_{3}^{(2)}= & \frac{1}{w_{0}}\left\{\int_{s_{2}}^{s_{3}} \frac{s \mathrm{~d} s}{\sqrt{\left(s-s_{1}\right)\left(s-s_{2}\right)\left(s_{3}-s\right)\left(s_{4}-s\right)}}+\int_{t_{2}}^{t_{3}} \frac{t \mathrm{~d} t}{\sqrt{\left(t-t_{1}\right)\left(t-t_{2}\right)\left(t_{3}-t\right)\left(t_{4}-t\right)}}\right. \\
& \left.+\int_{u_{2}}^{u_{3}} \frac{u \mathrm{~d} u}{\sqrt{\left(u-u_{1}\right)\left(u-u_{2}\right)\left(u_{3}-u\right)\left(u_{4}-u\right)}}\right\}
\end{aligned}
$$

where the roots $t_{i}$ and $u_{i}$ can be obtained from $s_{i}$ given in Eq. (1.6) by proper permutation of the masses $m_{i}$. Each of the integrals involved in Eq. (5.8) can be expressed in terms of Jacobian $Z$ function (see in Appendix). For example,

$$
\int_{s_{2}}^{s_{3}} \frac{s \mathrm{~d} s}{\sqrt{\left(s-s_{1}\right)\left(s-s_{2}\right)\left(s_{3}-s\right)\left(s_{4}-s\right)}}=\frac{\sin \varphi_{3}}{\sin \varphi_{1} \sin \varphi_{2}} K(k)-K(k) Z\left(\varphi_{3}, k\right),
$$

where $\varphi_{i}$ are nothing but the three angles defined in Eqs. (4.20)-(4.21). Comparing the resulting expression with the original result (5.7) we obtain a very useful relation between the three $Z\left(\varphi_{i}, k\right)$ functions,

$$
Z\left(\varphi_{1}, k\right)+Z\left(\varphi_{2}, k\right)+Z\left(\varphi_{3}, k\right)=k^{2} \sin \varphi_{1} \sin \varphi_{2} \sin \varphi_{3} .
$$

Let us now consider the four-dimensional integral $I_{3}^{(4)} \equiv I_{3}$, namely, its representation (1.5). A useful observation is that the result would be simpler if we managed to get rid of $s$ in the denominator. In particular, it would contain just one elliptic integral $\Pi$, rather than two. How are we to eliminate $s$ in the denominator? Again, using the $\delta$ function in Eq. (5.1), we can insert $1=(s+t+u) / w_{0}$ in the integrand, and then consider the three resulting terms (with $s, t$ and $u$ ) separately. For the term with $s$, we perform the $t$ and $u$ integrations and arrive at the same integral as in Eq. (1.5), but without $s$ in the denominator. In two other integrals, we just integrate in a different order, leaving as the last one the $t$ or $u$ integration, respectively. In this way, we obtain for the integral (5.1)

$$
\begin{gathered}
\frac{\pi^{2}}{4 p^{2} w_{0}} \iiint \mathrm{d} s \mathrm{~d} t \mathrm{~d} u(s+t+u) \delta\left(s+t+u-w_{0}\right) \theta\left(s t u-s t_{0} u_{0}-s_{0} t u_{0}-s_{0} t_{0} u+2 s_{0} t_{0} u_{0}\right) \\
=\frac{\pi^{2}}{4 p^{2} w_{0}}\left\{\int_{s_{2}}^{s_{3}} \mathrm{~d} s \sqrt{\left(s-s_{1}\right)\left(s-s_{2}\right)\left(s_{3}-s\right)\left(s_{4}-s\right)}+\int_{t_{2}}^{t_{3}} \mathrm{~d} t \sqrt{\left(t-t_{1}\right)\left(t-t_{2}\right)\left(t_{3}-t\right)\left(t_{4}-t\right)}\right. \\
\left.+\int_{u_{2}}^{u_{3}} \mathrm{~d} u \sqrt{\left(u-u_{1}\right)\left(u-u_{2}\right)\left(u_{3}-u\right)\left(u_{4}-u\right)}\right\}
\end{gathered}
$$


where, as before, the roots $t_{i}$ and $u_{i}$ can be obtained from $s_{i}$ given in Eq. (1.6) by permutation of the masses.

Using the formulae given in Ref. 24] along with Eqs. (A.8) and (A.11), the $s$-integral in Eq. (5.11) can be calculated in terms of a Jacobian $Z$ function (see Appendix),

$$
\begin{aligned}
\int_{s_{2}}^{s_{3}} \mathrm{~d} s & \sqrt{\left(s-s_{1}\right)\left(s-s_{2}\right)\left(s_{3}-s\right)\left(s_{4}-s\right)} \\
= & \sqrt{Q_{+}}\left\{2\left(p^{2} m_{3}^{2}-m_{1}^{2} m_{2}^{2}\right) K(k) \frac{Z\left(\varphi_{3}, k\right)}{\sin \varphi_{3}}+\frac{1}{6} Q_{-} K(k)\right. \\
& \left.+\frac{1}{6}\left[\left(p^{2}-m_{1}^{2}-m_{2}^{2}+m_{3}^{2}\right)^{2}+8\left(p^{2} m_{3}^{2}+m_{1}^{2} m_{2}^{2}\right)\right][E(k)-K(k)]\right\},
\end{aligned}
$$

where $\varphi_{3}$ is one of the three angles defined in Eqs. (4.20)-4.21).

Collecting the results for all three integrals and using the relation (5.10), we arrive at the symmetric result

$$
\begin{aligned}
I_{3}= & \frac{\pi^{2}}{8 p^{2}}\left\{\sqrt{Q_{+}}\left(p^{2}+m_{1}^{2}+m_{2}^{2}+m_{3}^{2}\right)[E(k)-K(k)]\right. \\
& \left.+Q_{+} K(k)\left[\frac{Z\left(\varphi_{1}, k\right)}{\sin ^{2} \varphi_{1}}+\frac{Z\left(\varphi_{2}, k\right)}{\sin ^{2} \varphi_{2}}+\frac{Z\left(\varphi_{3}, k\right)}{\sin ^{2} \varphi_{3}}\right]\right\} .
\end{aligned}
$$

This symmetric result can also be presented in terms of the elliptic integrals $\Pi$, using (see in [24])

$$
K(k) Z\left(\varphi_{i}, k\right)=\cot \varphi_{i} \sqrt{1-k^{2} \sin ^{2} \varphi_{i}}\left[\Pi\left(k^{2} \sin ^{2} \varphi_{i}, k\right)-K(k)\right] .
$$

In principle, one can also derive the result (5.13) directly from the non-symmetric representation (1.7) (see in Ref. 25]), in a tedious way relying on the use of several relations collected in the Appendix, including the addition formula (A.9) for Jacobi $Z$ functions.

Worth noting is that in a similar way one can obtain results for higher even dimensions $D$. For instance, in six dimensions we get

$$
\begin{aligned}
I_{3}^{(6)}= & \frac{\pi^{4}}{144 p^{4}}\left\{\frac { Q _ { + } ^ { 1 / 2 } } { 2 0 } [ E ( k ) - K ( k ) ] \left[192\left(p^{8}+m_{1}^{8}+m_{2}^{8}+m_{3}^{8}\right)-112\left(p^{4}+m_{1}^{4}+m_{2}^{4}+m_{3}^{4}\right)^{2}\right.\right. \\
& -6\left(p^{2}+m_{1}^{2}+m_{2}^{2}+m_{3}^{2}\right)^{4}-156\left(p^{6}+m_{1}^{6}+m_{2}^{6}+m_{3}^{6}\right)\left(p^{2}+m_{1}^{2}+m_{2}^{2}+m_{3}^{2}\right) \\
& \left.+83\left(p^{4}+m_{1}^{4}+m_{2}^{4}+m_{3}^{4}\right)\left(p^{2}+m_{1}^{2}+m_{2}^{2}+m_{3}^{2}\right)^{2}\right] \\
& +\frac{1}{40} Q_{-} Q_{+}^{1 / 2} K(k)\left[3\left(p^{2}+m_{1}^{2}+m_{2}^{2}+m_{3}^{2}\right)^{2}-16\left(p^{4}+m_{1}^{4}+m_{2}^{4}+m_{3}^{4}\right)\right] \\
& +\frac{3}{4} \frac{Q_{+}^{5 / 2} K(k)}{\sin \varphi_{1} \sin \varphi_{2} \sin \varphi_{3}}\left[\frac{Z\left(\varphi_{1}, k\right)}{\sin ^{2} \varphi_{1}}+\frac{Z\left(\varphi_{2}, k\right)}{\sin ^{2} \varphi_{2}}+\frac{Z\left(\varphi_{3}, k\right)}{\sin ^{2} \varphi_{3}}\right]
\end{aligned}
$$




$$
\left.-\frac{3}{8} Q_{+}^{2}\left(p^{2}+m_{1}^{2}+m_{2}^{2}+m_{3}^{2}\right) K(k)\left[\frac{Z\left(\varphi_{1}, k\right)}{\sin ^{4} \varphi_{1}}+\frac{Z\left(\varphi_{2}, k\right)}{\sin ^{4} \varphi_{2}}+\frac{Z\left(\varphi_{3}, k\right)}{\sin ^{4} \varphi_{3}}\right]\right\} .
$$

As an alternative way to obtain results for higher values of $D$, the approach of the paper [9] may be used.

In the equal-mass case,

$$
\varphi_{1}=\varphi_{2}=\varphi_{3} \equiv \varphi_{\mathrm{eq}}, \quad \sin \varphi_{\mathrm{eq}}=\frac{\sqrt{(p-m)(p+3 m)}}{p+m}, \quad \cos \varphi_{\mathrm{eq}}=\frac{2 m}{p+m} .
$$

Here, using Eq. (5.10) we get

$$
Z\left(\varphi_{\mathrm{eq}}, k_{\mathrm{eq}}\right)=\frac{1}{3} k_{\text {eq }}^{2} \sin ^{3} \varphi_{\mathrm{eq}},
$$

with $k_{\text {eq }}$ defined in Eq. (1.12). In this way, we reproduce Eq. (1.11), whereas for $D=6$ Eq. (5.15) yields

$$
\begin{aligned}
\frac{\pi^{4}}{2880 p^{4}} \sqrt{(p-m)^{3}(p+3 m)}\{ & \left(p^{4}-9 m^{4}\right)\left(p^{4}-42 p^{2} m^{2}+9 m^{4}\right)\left[E\left(k_{\text {eq }}\right)-K\left(k_{\text {eq }}\right)\right] \\
& \left.+(p+m)^{3}(p-3 m)\left(p^{4}-36 p^{2} m^{2}+27 m^{4}\right) K\left(k_{\text {eq }}\right)\right\} .
\end{aligned}
$$

We note that Eq. (5.17) yields a reduction formula of $Z(\varphi, k)$, for a special case when

$$
k=\frac{\sqrt{1-2 \cos \varphi}}{\sin \varphi(1-\cos \varphi)} .
$$

Another interesting limit corresponds to the case when one of the masses vanishes (for example, $\left.m_{3} \rightarrow 0\right)$. This corresponds to the case $k \rightarrow 1$, when $E(k)$ is finite $(E(1)=1)$ whereas $K(k)$ develops logarithmic singularity. At $m_{3}=0, \cos \varphi_{3}<0$ and $\varphi_{3}>\pi / 2$, so that we need to use Eq. (A.7). Using equations listed in Ref. [24], we get

$$
\lim _{k \rightarrow 1}\{K(k)[ \pm Z(\varphi, k)-\sin \varphi]\}=-\frac{1}{2} \ln \left(\frac{1+\sin \varphi}{1-\sin \varphi}\right),
$$

where plus or minus should be used for $\varphi<\pi / 2$ or $\varphi>\pi / 2$, respectively. Let us consider Eq. (5.13). Using Eqs. (5.19) and (4.21) we see that singular terms containing $K(k)$ cancel, and we arrive at the following result:

$$
\begin{aligned}
\lim _{m_{3} \rightarrow 0} I_{3}=\frac{\pi^{2}}{8 p^{2}}\left\{\sqrt{Q_{+}}\left(p^{2}+m_{1}^{2}+m_{2}^{2}\right)+\frac{1}{2}\left(p^{2}-m_{1}^{2}-m_{2}^{2}\right)^{2} \ln \left(\frac{p^{2}-m_{1}^{2}-m_{2}^{2}+\sqrt{Q_{+}}}{p^{2}-m_{1}^{2}-m_{2}^{2}-\sqrt{Q_{+}}}\right)\right. \\
-\frac{1}{2}\left(p^{2}+m_{1}^{2}-m_{2}^{2}\right)^{2} \ln \left(\frac{p^{2}+m_{1}^{2}-m_{2}^{2}+\sqrt{Q_{+}}}{p^{2}+m_{1}^{2}-m_{2}^{2}-\sqrt{Q_{+}}}\right) \\
\left.-\frac{1}{2}\left(p^{2}-m_{1}^{2}+m_{2}^{2}\right)^{2} \ln \left(\frac{p^{2}-m_{1}^{2}+m_{2}^{2}+\sqrt{Q_{+}}}{p^{2}-m_{1}^{2}+m_{2}^{2}-\sqrt{Q_{+}}}\right)\right\}
\end{aligned}
$$


where $Q_{+}=\lambda\left(p^{2}, m_{1}^{2}, m_{2}^{2}\right)$ in this limit. It is easy to check that this expression is equivalent to known results (see, e.g., in Refs. [3, 8]). The advantage of our approach is that the symmetry with respect to any of the remaining masses is always explicit, whereas nonsymmetric expressions like Eq. (1.7) lead to the answers which are not explicitly symmetric (cf. Eq. (57) of Ref. [8]).

\section{Conclusion}

We have considered several representations for the three-particle phase space, exploring their symmetry properties and geometrical meaning. It was shown that the angles $\varphi_{i}$ defined in Eqs. (4.20) - (4.21) are convenient to describe the results for the three-particle phase-space integral $I_{3}$. In terms of the Jacobian $Z$ function (related to the elliptic integral $\Pi$ through Eq.(5.14) ), the result for $I_{3}$ in four dimensions is given in Eq. (5.13). It is very compact and explicitly symmetric with respect to all masses $m_{i}$. Note that the three zeta functions $Z\left(\varphi_{i}, k\right)$ are connected through the relation (5.10). This relation can be obtained by comparing the representation (5.7) for two-dimensional integral $I_{3}^{(2)}$ with another representation obtained by using the delta function properties. We have

also considered the six-dimensional case. The result for $I_{3}^{(6)}$ is given in Eq. (5.15), also expressed in terms of $Z\left(\varphi_{i}, k\right)$.

Acknowledgements. We are pleased to acknowledge financial support from the Australian Research Council under grant number A00000780. Partical support from the Deutsche Forschungsgemeinschaft (A. D.) is also acknowledged.

\section{Appendix: Elliptic integrals}

The normal elliptic integrals of the first and second kind are defined as

$$
\begin{aligned}
& F(\varphi, k)=\int_{0}^{\sin \varphi} \frac{\mathrm{d} t}{\sqrt{\left(1-t^{2}\right)\left(1-k^{2} t^{2}\right)}}=\int_{0}^{\varphi} \frac{\mathrm{d} \psi}{\sqrt{1-k^{2} \sin ^{2} \psi}} \\
& E(\varphi, k)=\int_{0}^{\sin \varphi} \mathrm{d} t \sqrt{\frac{1-k^{2} t^{2}}{1-t^{2}}}=\int_{0}^{\varphi} \mathrm{d} \psi \sqrt{1-k^{2} \sin ^{2} \psi} .
\end{aligned}
$$

At $\varphi=\pi / 2$ we get the complete elliptic integrals,

$$
\begin{aligned}
& K(k)=F\left(\frac{\pi}{2}, k\right)=\int_{0}^{1} \frac{\mathrm{d} t}{\sqrt{\left(1-t^{2}\right)\left(1-k^{2} t^{2}\right)}}=\frac{\pi}{2}{ }_{2} F_{1}\left(\begin{array}{cc}
-\frac{1}{2}, & \frac{1}{2} \\
1
\end{array} \mid k^{2}\right), \\
& E(k)=E\left(\frac{\pi}{2}, k\right)=\int_{0}^{1} \mathrm{~d} t \sqrt{\frac{1-k^{2} t^{2}}{1-t^{2}}}=\frac{\pi}{2}{ }_{2} F_{1}\left(\begin{array}{c}
\frac{1}{2}, \\
1
\end{array}\left|\frac{1}{2}\right| k^{2}\right),
\end{aligned}
$$




$$
\Pi(c, k)=\int_{0}^{1} \frac{\mathrm{d} t}{\left(1-c t^{2}\right) \sqrt{\left(1-t^{2}\right)\left(1-k^{2} t^{2}\right)}}=\frac{\pi}{2} F_{1}\left(\frac{1}{2} ; 1, \frac{1}{2} ; 1 \mid c, k^{2}\right),
$$

where $F_{1}$ is the Appell hypergeometric function of two arguments.

The Jacobian zeta function, $Z(\beta, k)$, is defined through

$$
K(k) Z(\beta, k)=K(k) E(\beta, k)-E(k) F(\beta, k) .
$$

We will assume that $0 \leq k<1$. (In the limit $k \rightarrow 1, F(\beta, k)$ and $K(k)$ are singular.) From the definition (A.6) it is obvious that $Z\left(\frac{\pi}{2}, k\right)=0$. Moreover, using symmetry properties of $E(\beta, k)$ and $F(\beta, k)$, we get

$$
Z\left(\frac{\pi}{2}+\delta, k\right)=-Z\left(\frac{\pi}{2}-\delta, k\right) .
$$

To represent the elliptic functions $\Pi\left(\alpha_{i}, k\right)$ occurring in Eq. (1.7) in terms of $Z$ functions, we can use

$$
\Pi\left(\alpha_{i}^{2}, k\right)=K(k)+\frac{\alpha_{i} K(k) Z\left(\beta_{i}, k\right)}{\sqrt{\left(1-\alpha_{i}^{2}\right)\left(k^{2}-\alpha_{i}^{2}\right)}},
$$

with $\beta_{i}=\arcsin \left(\alpha_{i} / k\right)$. Eq. (A.8) corresponds to case III on p. 229 of [24], when $0 \leq$ $\alpha_{i}^{2}<k^{2}$.

The following addition formulae from Ref. [24] (p. 34, Eq. (142.01)) are needed:

$$
Z\left(\beta_{1}, k\right) \pm Z\left(\beta_{2}, k\right)=Z\left(\varphi_{ \pm}, k\right) \pm k^{2} \sin \beta_{1} \sin \beta_{2} \sin \varphi_{ \pm},
$$

where the angles

$$
\varphi_{ \pm}=2 \arctan \left[\frac{\sin \beta_{1} \sqrt{1-k^{2} \sin ^{2} \beta_{2}} \pm \sin \beta_{2} \sqrt{1-k^{2} \sin ^{2} \beta_{1}}}{\cos \beta_{1}+\cos \beta_{2}}\right] .
$$

In fact, the angles $\varphi_{-}$and $\varphi_{+}$correspond to the angles $\varphi_{1}$ and $\varphi_{2}$ (see Eqs. (4.20)-(4.21)), respectively. Moreover, using the same addition formula (A.9) with $\beta_{1,2}$ substituted by $\varphi_{1,2}$, we get the symmetric connection (5.10) between $Z\left(\varphi_{i}, k\right)(i=1,2,3)$.

To derive the result given in Eq. (5.12), one can use the integral tables of Ref. [24], along with the following relation:

$$
2 Z\left(\beta_{1}, k\right)=-Z\left(\varphi_{3}, k\right)+\frac{2 k^{2} \sin ^{3} \beta_{1} \cos \beta_{1} \sqrt{1-k^{2} \sin ^{2} \beta_{1}}}{1-k^{2} \sin ^{4} \beta_{1}} .
$$

It corresponds to the last two lines of Eq. (141.01) on p. 33 of Ref. [24], where $\varphi \leftrightarrow \pi-\varphi_{3}$. 


\section{References}

[1] R.H. Dalitz, Phil. Mag. 44, 1068 (1953).

[2] E. Fabri, Nuovo. Cim. 11, 479 (1954).

[3] B. Almgren, Arkiv för Physik 38, 161 (1968).

[4] A. Bashir, R. Delbourgo and M.L. Roberts, J. Math. Phys. 42, 5553 (2001).

[5] R. Hagedorn, Nuovo Cim. 25, 1017 (1962).

[6] R. Delbourgo and M.L. Roberts, J. Phys. A36, 1719 (2003).

[7] G. Källen, Elementary Particle Physics, Addison-Wesley, Reading (MA), 1964.

[8] S. Bauberger, F. Berends, M. Böhm and M. Buza, Nucl. Phys. B434, 383 (1995).

[9] O.V. Tarasov, Nucl. Phys. B502, 455 (1997).

[10] A.I. Davydychev and V.A. Smirnov, Nucl. Phys. B554, 391 (1999).

[11] A. Aste and D. Trautmann, hep-th/0309059

[12] S. Mandelstam, Proc. Roy. Soc. Lond. A233, 248 (1955);

Phys. Rev. 115, 1741 (1959).

[13] T.W.B. Kibble, Phys. Rev. 117, 1159 (1960).

[14] B.A. Arbuzov, E.E. Boos, S.S. Kurennoy and K.Sh. Turashvili, Yad. Fiz. 44, 1565 (1986) [Sov. J. Nucl. Phys. 44, 1017 (1986)].

[15] E. Mendels, Nuovo Cim. A45, 87 (1978); J. Math. Phys. 43, 3011 (2002).

[16] A.P. Prudnikov, Yu.A. Brychkov and O.I. Marichev, Integrals and Series, Vol. 2, New York, Gordon and Breach, 1990.

[17] A.I. Davydychev, Phys. Rev. D61, 087701 (2000).

[18] E. Byckling and K. Kajantie, Particle Kinematics, John Wiley and Sons, 1973.

[19] A.I. Davydychev and R. Delbourgo, J. Math. Phys. 39, 4299 (1998).

[20] F.A. Berends, A.I. Davydychev and N.I. Ussyukina, Phys. Lett. B426, 95 (1998).

[21] A.K. Rajantie, Nucl. Phys. B480, 729 (1996); B513, 761(E) (1998).

[22] S. Groote, J.G. Körner and A.A. Pivovarov, Nucl. Phys. B542, 515 (1999);

S. Groote and A.A. Pivovarov, Nucl. Phys. B580, 459 (2000). 
[23] M.L. Roberts, Ph.D. thesis, University of Tasmania, Hobart, 2003.

[24] P.F. Byrd and M.D. Friedman, Handbook of Elliptic Integrals for Engineers and Physicists, Springer, 1954.

[25] A.I. Davydychev and R. Delbourgo, Proc. 15th Biennial Congress of the Australian Institute of Physics, Sydney, Australia, July 2002 (Causal Productions, Adelaide, 2002), p. 144 (hep-th/0209233) 